
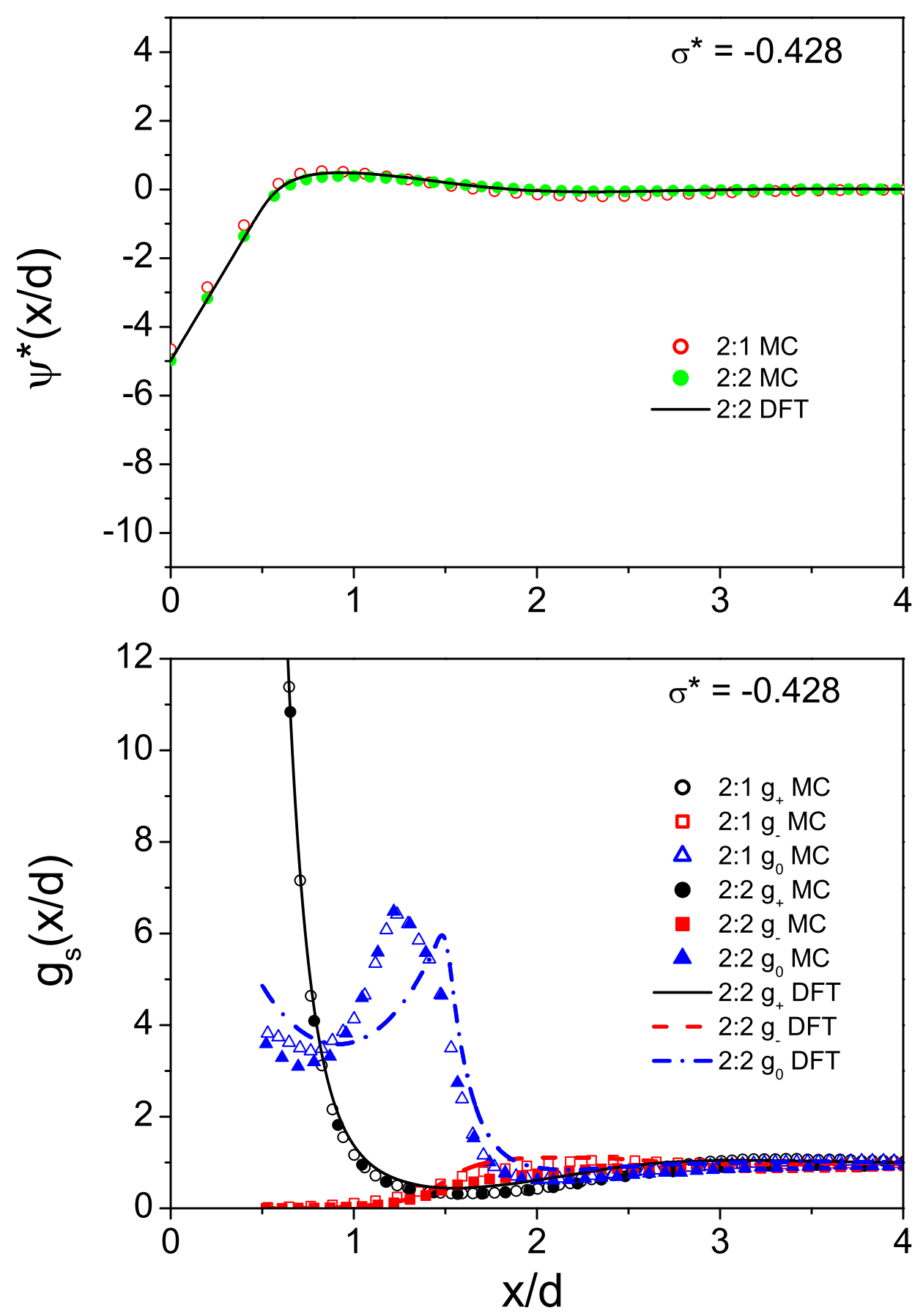

Mean electrostatic potential (upper panel) and electrode -- ion singlet distributions (lower panel) for a 2:2 valency dimer electrolyte model of the planar electric double layer. 


\title{
Structure of an electric double layer containing a 2:2 valency dimer electrolyte
}

\author{
Whasington Silvestre-Alcantara ${ }^{a}$, Douglas Henderson ${ }^{b}$, Jianzhong $\mathrm{Wu}^{c}$ \\ Monika Kaja $^{d}$, Stanisław Lamperski ${ }^{d}$, and Lutful Bari Bhuiyan ${ }^{a *}$
}

November 22, 2014

${ }^{a}$ Laboratory of Theoretical Physics, Department of Physics, University of Puerto Rico,
San Juan, PR 00936-8377, USA
${ }^{b}$ Department of Chemistry and Biochemistry, Brigham Young University, Provo, UT
84602-5700, USA
${ }^{c}$ Department of Chemical and Environmental Engineering, University of California,
Riverside, CA 92521-0425, USA
${ }^{d}$ Department of Physical Chemistry, Adam Mickiewicz University in Poznań, Umul-
towska 89b, 61-614 Poznań, Poland
${ }^{*}$ Corresponding Author: telephone+1 (787) 764 0000/extn 3579, e-mail beena@ beena.uprrp.edu 


\begin{abstract}
The structure of a planar electric double layer formed by a 2:2 valency dimer electrolyte in the vicinity of a uniformly charged planar hard electrode is investigated using density functional theory and Monte Carlo simulations. The dimer electrolyte consists of a mixture of charged divalent dimers and charged divalent monomers in a dielectric continuum. A dimer is constructed by two tangentially tethered rigid spheres, one of which is divalent and positively charged and the other neutral, whereas the monomer is a divalent and negatively charged rigid sphere. The density functional theory reproduces well the simulation results for (i) the singlet distributions of the various ion species with respect to the electrode, and (ii) the mean electrostatic potential. Comparison with earlier results for a 2:1/1:2 dimer electrolyte shows that the double layer structure is similar when the counterion has the same valency.
\end{abstract}




\section{Introduction}

We are pleased to dedicate this article to our friend and collaborator Darsh Wasan, to help celebrate his distinguished tenure as Editor-in-Chief of this journal. To be an exemplary editor is commendable. To combine this with a highly successful research career is exceptional but to combine both of these with a successful vice-presidency of an important university is truly outstanding.

An electric double layer is the name given to an inhomogeneous fluid system formed by a charged liquid (viz., an electrolyte, an ionic liquid, or a molten salt) in the vicinity of an electrode. Although the classical theory of the phenomenon [1-3] has been around for over a hundred years, the increasing relevance of the double layer in, for example, functioning of ion channels, DNA sequencing, and designing super-capacitors and lithium-ion batteries has continued to attract interest (see for example refs.[4,5]) and requires a more accurate and modern theory.

The underlying model of the classical Gouy-Chapman-Stern (GCS) [1-3] theory of the electric double layer is the primitive model (PM) where the charged liquid consists of charged hard spheres in a uniform dielectric continuum (the solvent) characterized by its relative permittivity. In the restrictive case of the spheres having the same diameter, it is the restricted primitive model (RPM). The RPM and, to a lesser extent the PM double layers have been extensively studied for the better part of the last four decades through formal statistical mechanical approaches and numerical simulations and a great deal of knowledge about such systems has been acquired (see for example, the reviews $[6,7])$. The simulations have further established that the mean-field nature of the classical picture, for instance, neglect of the interionic correlations and ionic exclusion volume effects, is less than adequate in most situations.

In recent years experiments especially those involving differential capacitance $\left(C_{D}\right)$ measurements have begun to expose the limitations of the PM double layer. For example, ionic liquid double layers manifest a variety of $C_{D}$ versus electrode surface charge density $\sigma$ behaviour [8-11], which can only be understood at best in rather broad terms using the RPM or the PM [12-23]. The primitive models necessarily introduce a shape 
symmetry in the constituent species of an electrolyte, which is probably not very realistic in the natural world. On the other hand, less simple models that have built-in asymmetry in ionic shape and/or size, molecules with off-centre charges might prove more useful in interpreting the experimental capacitance trends. By the same token complex models may prove too demanding theoretically. So the challenge is to be able to postulate more realistic models beyond the PM that are also amenable to theoretical treatment and/or numerical simulations. One such candidate is the dimer model of the electrolyte. Here, the cation is a dimer consisting of two tangentially tethered hard spheres one of which is positively charged and the other neutral, while the anion is a monomer, which is a negatively charged hard sphere. A related model is the trimer model with three touching, linearly aligned hard spheres with an end sphere being charged. Simulation studies on such models have been reported by Fedorov and Kornyshev [24], and Fedorov et al. [25], while the density functional theory (DFT) on the dimer model model has been applied by Fedorov et al. [25], Wu et al. [26], Henderson and Wu [27], and Henderson et al. [28]. There is some practical relevance of the dimer model in that it can mimic the alkyl cation of some ionic liquids and has been used to represent tetraethylammonium (an ionic liquid) in a pore [29]. Furthermore, molecular dynamics (MD) simulation techniques have been performed on ionic liquids like imidazolium based on a trimer model consisting of a spherical anion and a cation formed by three coplanar connected spheres in the form of a triangle [30].

In a series of papers some of us have explored the dimer electrolyte model of the double layer using Monte Carlo (MC) simulations and/or the DFT in both ionic liquid regime [31] and electrolyte solution regime [32,33] for 1:1 valency systems. A more recent later work [34] delves into 2:1/1:2 asymmetric valency systems. The principal findings have been (i) significant differences in structure and capacitances relative to that with the spherically symmetric PM, (ii) a richer double layer structure when the counterions are non-spherical, (iii) asymmetry in the $C_{D}$ versus $\sigma$ plots that still show minimum-maximum transition at high salt concentrations, and (iv) similarity in double layer structure between systems where the counterion has the same valency. In the present paper we will continue to look at the dimer model but now for higher 2:2 valency situations. The use of DFT ought to be of interest since higher and/or asymmetric valencies are always a more stringent test of a theory. 


\section{Model and Methods}

We have treated here the dimer model of the double layer in planar symmetry, that is, the electrode is a uniformly charged, planar hard surface. The constituent spheres of the dimer cation and also the monomer anion all have the same diameter $d$. A relative permittivity $\epsilon_{r}$ specifies the continuum dielectric (solvent). The model used is exactly the same as in the earlier studies [29-33] excepting that the valencies of the cation and the anion are now +2 and -2 , respectively. The MC simulation techniques and the DFT employed are the same as used in the previous papers [29, 31-34] and will not be repeated here.

\section{Results}

All of the results presented here, except for a couple of exceptions, pertain to $2^{+}: 2^{-}$ valency dimer electrolytes at temperature $T=298 \mathrm{~K}, \epsilon_{r}=78.5$ corresponding to a water-like solvent, and electrolyte concentration $c=1 \mathrm{~mol} / \mathrm{dm}^{3}$. The exceptions are two sets of MC simulations for $2^{+}: 1^{-}$and $1^{+}: 2^{-}$electrolytes, also at $1 \mathrm{~mol} / \mathrm{dm}^{3}$ and for a positive electrode, which were done for comparison purposes. In the rest of the paper we will refer to these electrolyte systems as $2: 2,2: 1$, and 1:2 systems with the understanding that the first index always refers to the valency of the dimer cation and the second index to the valency of the monomer anion. Following the usual custom, we will discuss the results in terms of the following reduced variables, viz., the reduced density

$$
\rho_{s}^{*}=\rho_{s} d^{3}
$$

where $\rho_{s}$ is the mean bulk number density of ion species $s$, the reduced surface charge density

$$
\sigma^{*}=\frac{\sigma d^{2}}{e}
$$

the reduced mean electrostatic potential

$$
\psi^{*}(x)=e \beta \psi(x),
$$


and the reduced temperature

$$
T^{*}=\frac{4 \pi \epsilon_{0} \epsilon_{r} d}{\left|Z_{+} Z_{-}\right| \beta e^{2}}
$$

In the above equations, $Z_{s}$ is the valency of species $s, e$ is the magnitude of the electronic charge, $\epsilon_{0}$ is the vacuum permittivity, and $\beta=1 /\left(k_{B} T\right)$ with $k_{B}$ the Boltzmann constant. The total reduced density is given by $\rho^{*}=\sum_{s} \rho_{s}^{*}$ with the summation extending over all the species. Also, $T^{*}=1 / \Gamma$, where $\Gamma$ is the plasma coupling parameter, which is a measure of the strength of the electrostatic interactions.

In Figs. 1 and 2 we show the electrode-ion singlet distribution profiles $g_{s}(x / d)$ (lower panels) and the reduced mean electrostatic potential $\psi^{*}(x / d)$ (upper panels) for the 2:2 electrolyte near a planar electrode with a negative surface charge. The corresponding 2:1 and 1:2 profiles from our earlier work [34] have been superimposed on Figs. 1 and 2, respectively, for comparison. It is noted that all the MC results are at $\sigma^{*}=-0.428$. This value of $\sigma^{*}$ for the 2:2 MC yielded a value of the reduced electrode potential $\psi^{*}(0)=-4.99$, which was used as an input in the DFT calculations, and which in turn gave the DFT value of $\sigma^{*}=-0.422$. Put in another way, the 2:2 DFT and $\mathrm{MC}$ results are at the same $\psi^{*}(0)$. We do this because the DFT numerical code that we employ uses $\psi^{*}(0)$ as an input parameter. Since our DFT result for the $\psi^{*}(0)$ versus $\sigma^{*}$ relation has proved very accurate [32], such a comparison is meaningful and avoids a tedious iteration to find the exact value of the DFT $\psi^{*}(0)$ for a given value of $\sigma^{*}$. We note further that the total reduced density $\rho^{*}=0.139,0.185$, and 0.231 for the cases $2: 2,2: 1$, and $1: 2$, respectively, while $T^{*}=0.149$ and 0.297 for the $2: 2$ and 2:1/1:2 cases, respectively. The first thing to notice in Fig. 1 is that the DFT lines follow the MC symbols for the ionic profiles very well. The correspondence between the DFT and MC neutral sphere profiles is also quite reasonable, although the DFT peak is shifted slightly towards a higher $x / d$ value. The agreement of the mean electrostatic potential profiles is nearly quantitative. The peak, albeit shallow, in the $\psi^{*}$ near the electrode signifies a charge reversal stemming from an over-screening of the electrode charge. Such agreement of the DFT and the MC is reminiscent of similar behaviour seen earlier for 1:1 valency systems [31-33]. A second point worth mentioning in this figure is the close proximity of MC 2:2 and 2:1 data points - systems with the same counterion valency. This point was noted in our earlier work on 2:1/1:2 valencies for the dimer electrolyte 
model [34]. This is further illustrated in Fig. 2 where the MC 2:2 is compared with the MC 1:2. Here the counterion valencies are different, and so are the various profiles. The $\psi^{*}(x / d)$ for the two systems are qualitatively different, for instance, in contrast to the non-monotonic nature of the $2: 2 \psi^{*}(x / d)$, that for the $1: 2$ is monotonic.

Figs. 3 and 4 display the profiles for a positive surface charge, $\sigma^{*}=0.428$. Here, the corresponding MC 2:2 $\psi^{*}(0)=4.32$, which was used to generate the DFT results with the DFT surface charge now being $\sigma^{*}=0.482$. Comparing the positive and negative charge situations, it appears that the DFT tends to overestimate $\sigma^{*}$ relative to the MC for a given positive electrode charge. In Figs. 3 and 4 we have included the (new) MC 1:2 and 2:1 results, respectively, obtained at the same surface charge density. The total reduced densities are the same as before, that is, $\rho^{*}=0.185$ for the $2: 1$ case and $\rho^{*}=$ 0.231 for the 1:2 case. Except for the peak in the distribution of the positive end of the dimers, the DFT profiles again follow the MC profiles closely. For the $\psi^{*}$ profile, the DFT result is also close to the MC. The minimum in this profile again suggests a charge reversal as seen before with the DFT minimum being slightly deeper than that for the MC. The relative behaviour of the 2:2 and 1:2 MC distributions follow the pattern seen in Figs. 1 and 2. The same counterion valency occurs in Fig. 3 where these profiles are similar, but are dissimilar in Fig. 4 where the counterion valencies are different.

Fig. 5 shows the density and the mean electrostatic profiles at a very low electrode charge density $\sigma^{*}=0.00960$. This value of $\sigma^{*}$ corresponds to the DFT input of $\psi^{*}(0)=$ 0 . The DFT $g_{s}$ show good agreements with the MC data for $x / d \geq 1$, but admittedly, at and near the contact region there is some discrepancy. Recently, Jiang et al. [35,36] have formulated a contact corrected version of the DFT for the planar double layer, which is consistent with the exact contact value relations of Henderson and Blum [37], and Henderson et al. [38]. Unfortunately, no such contact relation is yet available for the dimer double layer model. The $\psi^{*}(0)$ corresponding to the above $\sigma^{*}$ for the MC is 0.0195 , and as can be seen in the upper panel, the potential profiles are in good agreement.

In Figs. 6 and 7 we present the $\mathrm{MC} \psi^{*}(0)$ versus $\sigma^{*}$ plots and the orientation profile of the dimers, respectively. The 2:1 and 1:2 plots are from our previous work [34]. Even for the symmetric valency $2: 2$ case there is a finite, non-zero potential of zero charge (pzc), however small that occurs due to the anisotropic shape of the cation and 
consequently the relative asymmetry in both size and shape between the cation and the and integral $C_{I} \sim \sigma^{*} / \psi^{*}(0)$ ) behaviour in the systems. We note in passing that for positive $\sigma^{*}$ the 2:2 and 1:2 curves (counterion valency 2) are close, while for negative $\sigma^{*}$ the 2:2 and 2:1 curves (counterion valency 2 ) are close. These results are consistent with similar earlier observations [34]. To describe the average orientation of the dimers, in Fig. 7 we have plotted $<\cos (\theta)>$ as a function of $x / d$ for a negative surface charge. We define $\cos (\theta)=\left(x_{0}-x_{+}\right) / d$, where $x_{0}$ and $x_{+}$are the $x$-coordinates of the centres of the neutral and positively charged ends of a dimer, respectively. The 2:1 and 1:2 results are from the earlier work [34]. These profiles in conjunction with the neutral species' distribution in Figs. 1-4, describe this species' structure. The dimer orientation changes from being generally perpendicular to the electrode near the electrode to being more randomly oriented further away when bulk conditions are reached. We note again that the $2: 1$ plot is very similar to the $2: 2$ plot with the counterion valency being the same for the two cases.

Some comments on the comparative behaviour of the structural properties for the 1:1 dimer electrolyte [32] and the present 2:2 dimer electrolyte are in order. Both are valency symmetric but ionic shape anisotropic systems. The differences in structural pattern between the systems stem from the fact that the reduced temperature $T^{*}$ for the 1:1 case is four times that for the 2:2 case, which is to say that the 2:2 coupling is four times stronger than the 1:1 coupling. Universality arguments in terms of the reduced variables imply (from Eq. (4)) that the $2: 2$ system at $298 \mathrm{~K}$ is akin to a 1:1 system at $\sim 75 \mathrm{~K}$. Thus the stronger ion-ion and electrode-ion Coulomb interactions for the higher valencies dominate the structure in this situation. For example, for the same given concentration and surface charge density, the MC contact values of the counterion and coion profiles are greatly enhanced and depressed, respectively, in the 2:2 case relative to the 1:1 case. See for example, Fig. 2 of [32] and the Fig. 1 here. We have refrained though from showing the corresponding 1:1 results in Fig. 1 to avoid clutter. In either system, the neutral spheres of the dimers show more structure when the surface charge is negative and the dimers are close to the surface. The DFT is almost quantitative in reproducing the 1:1 MC profiles but somewhat less so vis-a-vis the 2:2 MC profiles. This is understandable since strong coupling regimes pose more 
of a theoretical challenge. Again for the 1:1 case, the dimer orientation profile changes more gradually to the random (bulk) state (cf. Fig. 4 of ref.[32]) relative to the 2:2 case (Fig. 7). In this sense, the 1:1 and the 1:2 orientation profiles would be similar and it is to be noted that they have the same counterion valency.

\section{Conclusions}

In summary, the major conclusions of this study are :

(a) the stronger interionic interactions and ion-wall interactions with 2:2 valencies lead to pronounced structure with manifest asymmetry due to the anisotropic ion shapes,

(b) the 2:2 density and potential profiles show substantial similarities with the corresponding 2:1 profiles when the electrode charge is negative and with 1:2 profiles when the electrode charge is positive. In either situation the counterion valencies are the same, which reinforces the notion that except for small electrode charge density, it is the electrode - counterion interaction that governs the double layer structure.

(c) the strength of the electrostatic coupling for the 2:2 electrolyte is four times that for the earlier studied 1:1 electrolyte [32], which leads to a relatively more compact double layer in the former situation, and

(d) in general the density functional theory reproduces well the simulation results for both the singlet distributions of the various species and the mean electrostatic potential. However, the performance of the theory is less satisfactory near a neutral surface. In particular, it overestimates the local charge segregation due to the asymmetry in ion shape.

A compelling rationale for treating models such as the present dimer model has been to impart some asymmetry in shape and size to the ions of the constituent electrolyte of the double layer and thereby bring the electrolyte more in line with many real charged fluids. The inherent asymmetry in the latter is clearly demonstrated in the experimental capacity measurements (see for example references [8-11]). The results of the present work in conjunction with our earlier studies [31-34] of the dimer electrolyte model of the planar double layer lend credence to the model's usefulness as an alternative to the ion shape symmetrical primitive models.

Variations of the dimer model can be contemplated, for example, when the two 
spheres (of different sizes) of the dimer are more fused into each other rather than merely touching tangentially. An extreme case would be when one sphere is completely immersed into the other leading to a charged sphere with an off-centre charge. Such projects are in progress.

\section{Acknowledgements}

MK and SL would like to acknowledge financial support from the Faculty of Chemistry, Adam Mickiewicz University in Poznań. This work was supported as part of the Fluid Interface Reactions, Structure and Transport (FIRST) Center, an Energy Frontier Research Center funded by the U.S. Department of Energy, Office of Science, Office of Basic Energy Sciences. 


\section{References}

[1] G. Gouy, J. Phys. (Paris) 9 (1910) 457.

[2] D. L. Chapman, Philos. Mag. 25 (1913) 475.

[3] O. Stern, Z. Elektrochem. 30 (1924) 508.

[4] C. A. Angell, Y. Ansari, Z. Zhao, Faraday Discuss. 154 (2012) 9.

[5] M. Galiński, A. Lewandowski, I. Stepniak, Electrochim. Acta 51 (2006) 5567.

[6] D. Henderson and D. Boda, Phys. Chem. Chem. Phys. 11 (2009) 3822.

[7] A. G. Cherstvy, Phys. Chem. Chem. Phys. 13, 9942 (2011).

[8] M. T. Alam, M. M. Islam, T. Okajima, T. Oshaka, Electrochem. Commun. 9 (2007) 2370 .

[9] M. M. Islam, M. T. Alam, T. Oshaka, J. Phys. Chem. C 112 (2008) 16568.

[10] M. T. Alam, M. M. Islam, T. Okajima, T. Oshaka, J. Phys. Chem. C 112 (2008) 16600 .

[11] M. T. Alam, M. M. Islam, T. Okajima, T. Oshaka, J. Phys. Chem. C 113 (2010) 6596.

[12] A. A. Koryshnev, J. Phys. Chem. B 111 (2007) 5545.

[13] M. S. Kilic, M. Z. Bazant, A. Ajdari, Phys. Rev. E: 75 (2007) 021502.

[14] Y. Han, S. Huang, T. Yan, J. Phys.:Condens. Matter 26 (2014) 284103.

[15] S. Lamperski, J. Kłos, J. Chem. Phys. 129 (2008) 164503.

[16] J. Kłos, S. Lamperski, J. Phys. Chem. C 114 (2010) 13329.

[17] J. Vatamanu, L. Cao, O. Borodin, G. D. Smith, Phys. Chem. Letts. 2 (2011) 2267.

[18] S. Lamperski, C. W. Outhwaite, and L. B. Bhuiyan, J. Phys. Chem. B 113 (2009) 8925; ibid 114 (2010) 3075. 
[19] C. W. Outhwaite, S. Lamperski, L. B. Bhuiyan, Mol. Phys. 109 (2011) 21.

[20] D. Henderson, S. Lamperski, C. W. Outhwaite, L. B. Bhuiyan, Collect. Czech. Chem. Comm. 75 (2010) 303.

[21] D. Henderson, S. Lamperski, Z. Jin, J. Wu J. Phys. Chem. B, 115 (2011) 12911.

[22] K. Breitspreecher, P. Košovan, C. Holm, J. Phys.: Condens. Matter 26 (2014) 284108.

[23] S. Lamperski, J. Sosnowska, L. B. Bhuiyan, D. Henderson, J. Chem. Phys., 140 (2014) 014704.

[24] M. V. Fedorov, A. A. Kornyshev, J. Phys. Chem. B 112 (2008) 11868.

[25] M. V. Fedorov, N. Georgi, A. A. Kornyshev, Electrochem. Commun. 12 (2010) 296.

[26] J. Wu, T. Jiang, D. Jiang, Z. Jin, D. Henderson, Soft Matter 7 (2011) 11222.

[27] D. Henderson and J. Wu, J. Phys. Chem. B 116 (2012) 2520.

[28] D. Henderson, S. Lamperski, Z. Jin, and J. Wu, J. Phys. Chem. B 115 (2011) 12911.

[29] D. Jiang, Z. Jin, D. Henderson, and J. Wu, J. Phys. Chem. Letters 3 (2012) 1727.

[30] K. Breitspreecher, P. Košovan, and C. Holm, J. Phys.:Condens. Matter 26 (2014) 284114.

[31] L. B. Bhuiyan, S. Lamperski, J. Wu, and D. Henderson, J. Phys. Chem. B 116, 10364 (2012).

[32] S. Lamperski, M. Kaja, L. B. Bhuiyan, J. Wu, and D. Henderson, J. Chem. Phys. 139, 054703 (2013).

[33] D. Henderson, S. Lamperski, L. B. Bhuiyan, J. Wu, J. Chem. Phys. 138 (2013) 144704. 
[34] M. Kaja, W. Silvestre-Alcantara, S. Lamperski, D. Henderson, L. B. Bhuiyan, Mol. Phys. DOI:10.1080/00268976.2014.968651.

[35] J. Jiang, D. Cao, D. Henderson, J. Wu, Phys. Chem. Chem. Phys. 16, (2014) 3934.

[36] J. Jiang, D. Cao, D. Henderson, J. Wu, J. Chem. Phys. 140, (2014) 044714.

[37] D. Henderson, L. Blum, J. Chem. Phys. 69, (1978) 5441.

[38] D. Henderson, L. Blum, and J. L. Lebowitz, J. Electroanal. Chem. 102, (1979) 315. 


\section{Figure Captions}

Figure 1 Reduced mean electrostatic potential $\psi^{*}$ (upper panel), electrode - ion singlet distributions $g_{s}$ (lower panel) for a 2:2 valency dimer electrolyte model of the planar electric double layer at concentration $c=1 \mathrm{~mol} / \mathrm{dm}^{3}$ (reduced density $\rho^{*}=0.139$ ) and reduced surface charge density $\sigma^{*}=-0.428 .2: 1 \mathrm{MC}$ results at the same $1 \mathrm{~mol} / \mathrm{dm}^{3}$ (reduced density $\rho^{*}=0.185$ ) from ref. [34] have been added for comparison purposes. The notations $g_{+}, g_{0}$, and $g_{-}$denote the singlet distributions for the positively charged ends of the dimers, the neutral ends of the dimers, and the negatively charged spheres, respectively. The rest of the legend is given within the figure.

Figure 2 Reduced mean electrostatic potential $\psi^{*}$ (upper panel), electrode - ion singlet distributions $g_{s}$ (lower panel) for 2:2 and 1:2 valency dimer electrolyte models of the planar double layer at concentration $c=1 \mathrm{~mol} / \mathrm{dm}^{3}$ and reduced surface charge density $\sigma^{*}=-0.428$. The 1:2 MC results are from ref. [34]. The notation as in Fig. 1 while the legend is given within the figure.

Figure 3 Reduced mean electrostatic potential $\psi^{*}$ (upper panel), electrode - ion singlet distributions $g_{s}$ (lower panel) for a 2:2 and 1:2 valency dimer electrolyte models of the planar electric double layer at concentration $c=1 \mathrm{~mol} / \mathrm{dm}^{3}$ and reduced surface charge density $\sigma^{*}=0.428$. The reduced densities $\rho^{*}=$ are 0.139 and 0.231 for the $2: 2$ and $1: 2$ cases, respectively. The notation as in Fig. 1 while the legend is given within the figure.

Figure 4 Reduced mean electrostatic potential $\psi^{*}$ (upper panel), electrode - ion singlet distributions $g_{s}$ (lower panel) for 2:2 and 2:1 valency dimer electrolyte models of the planar double layer at concentration $c=1 \mathrm{~mol} / \mathrm{dm}^{3}$ and reduced surface charge density $\sigma^{*}=-0.428$. The notation as in Fig. 1 while the legend is given within the figure.

Figure 5 Reduced mean electrostatic potential $\psi^{*}$ (upper panel), electrode - ion singlet distributions $g_{s}$ (lower panel) for a 2:2 valency dimer electrolyte model of the planar electric double layer at concentration $c=1 \mathrm{~mol} / \mathrm{dm}^{3}$ (reduced density $\rho^{*}=0.139$ ) and at a reduced surface charge density $\sigma^{*}=0.00960$. The notation as in Fig. 1 while the legend is given within the figure. 
Figure 6 Reduced mean electrostatic potential at contact $\psi^{*}(0)$ as a function of the reduced surface charge density $\sigma^{*}$ at concentration $c=1 \mathrm{~mol} / \mathrm{dm}^{3}$. The $2: 1$ and 1:2 MC results are from ref. [34]. The light line through the MC points acts as a visual aid. The legend is given within the figure.

Figure $7 \mathrm{MC}$ results for the average dimer orientation $<\cos (\theta)>$ as a function of $x / d$ for a 2:2 dimer electrolyte, a 2:1 dimer electrolyte, and a 1:2 dimer electrolyte at concentration $c=1 \mathrm{~mol} / \mathrm{dm}^{3}$ corresponding to reduced densities $\rho^{*}=0.139$ for $2: 2$, $\rho^{*}=0.185$ for $2: 1$, and $\rho^{*}=0.231$ for the $1: 2$ cases, respectively, and at surface charge density $\sigma^{*}=-0.428$. The legend is given within the figure. 

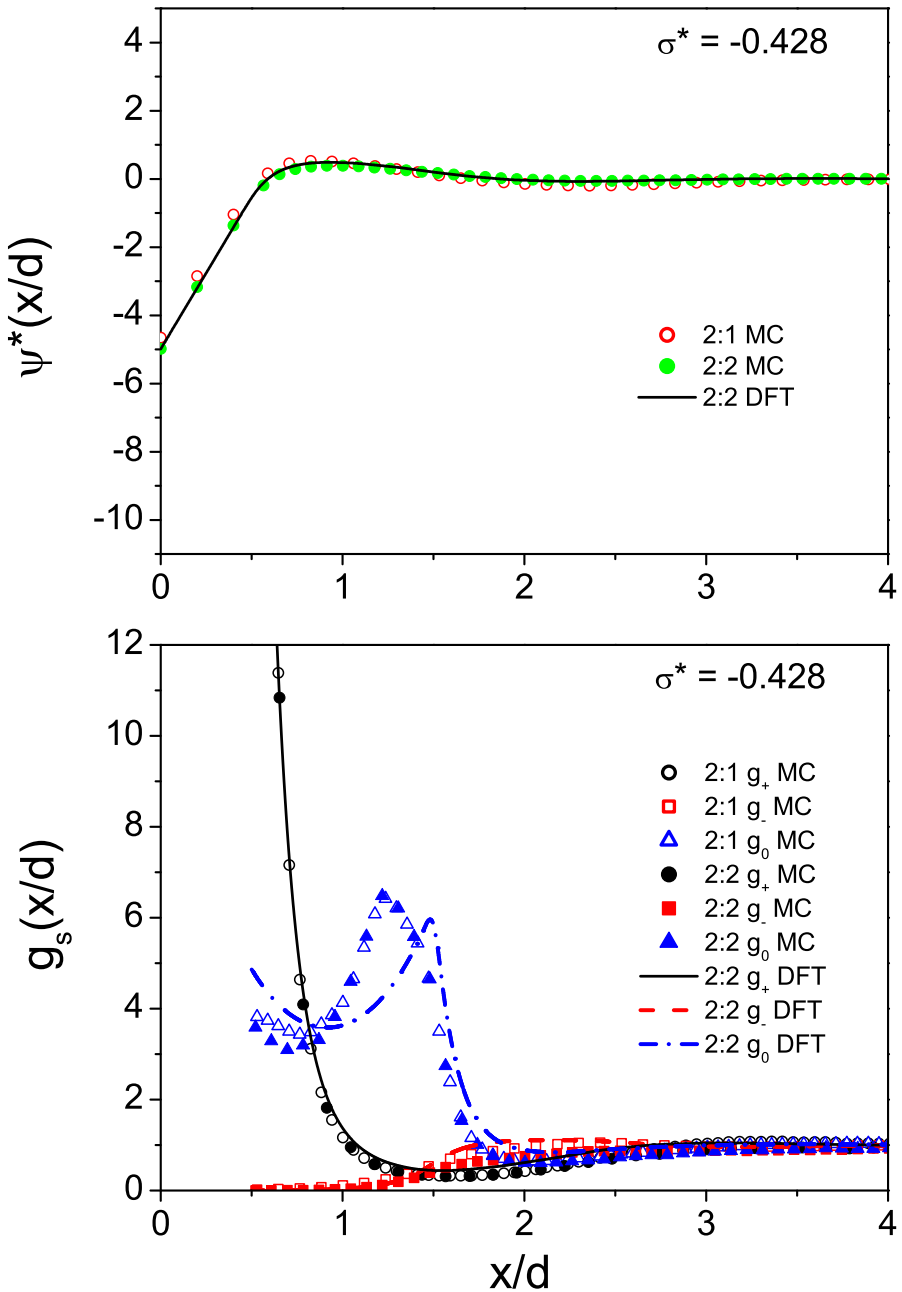

Figure 1

Figure 1: 

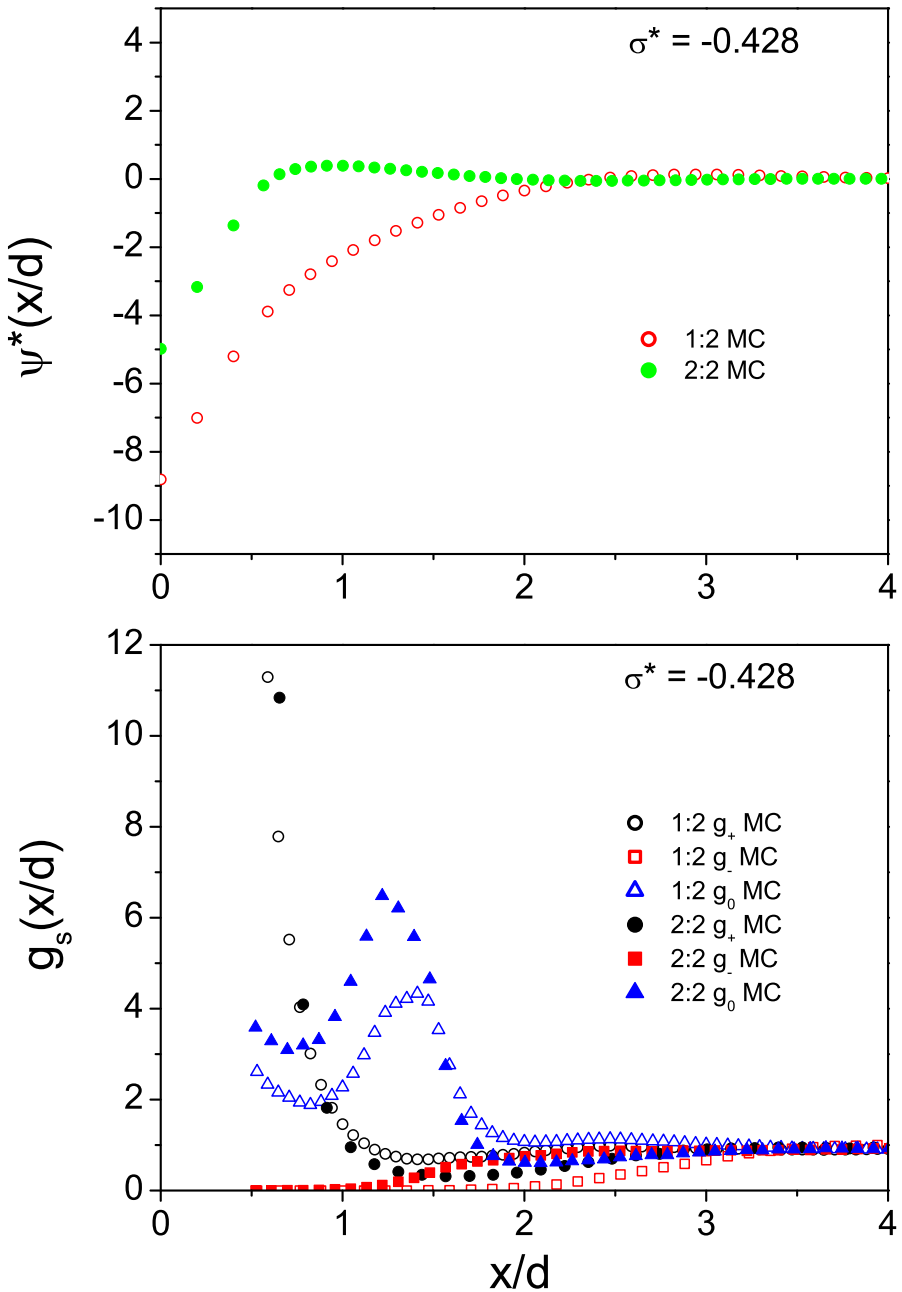

Figure 2

Figure 2: 

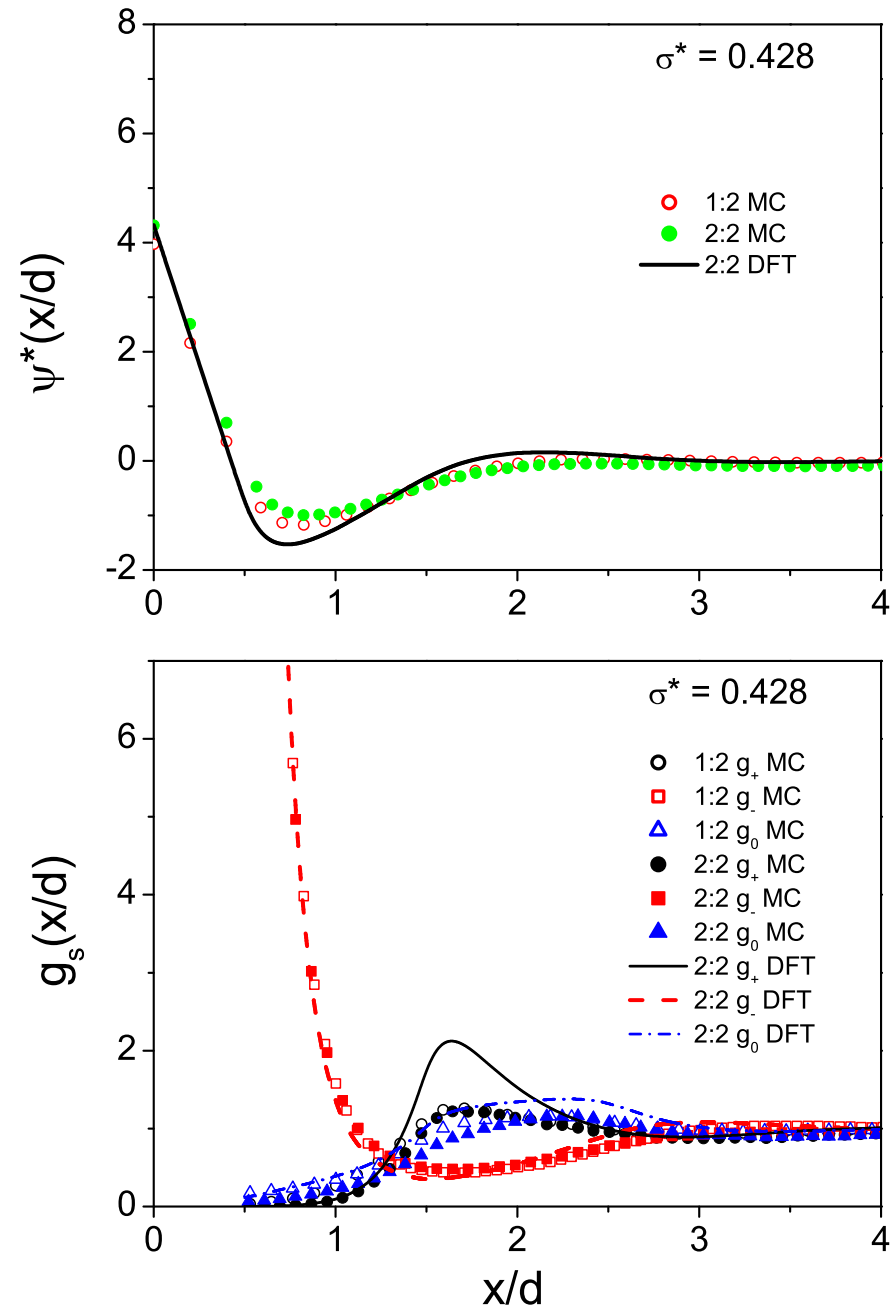

Figure 3

Figure 3: 

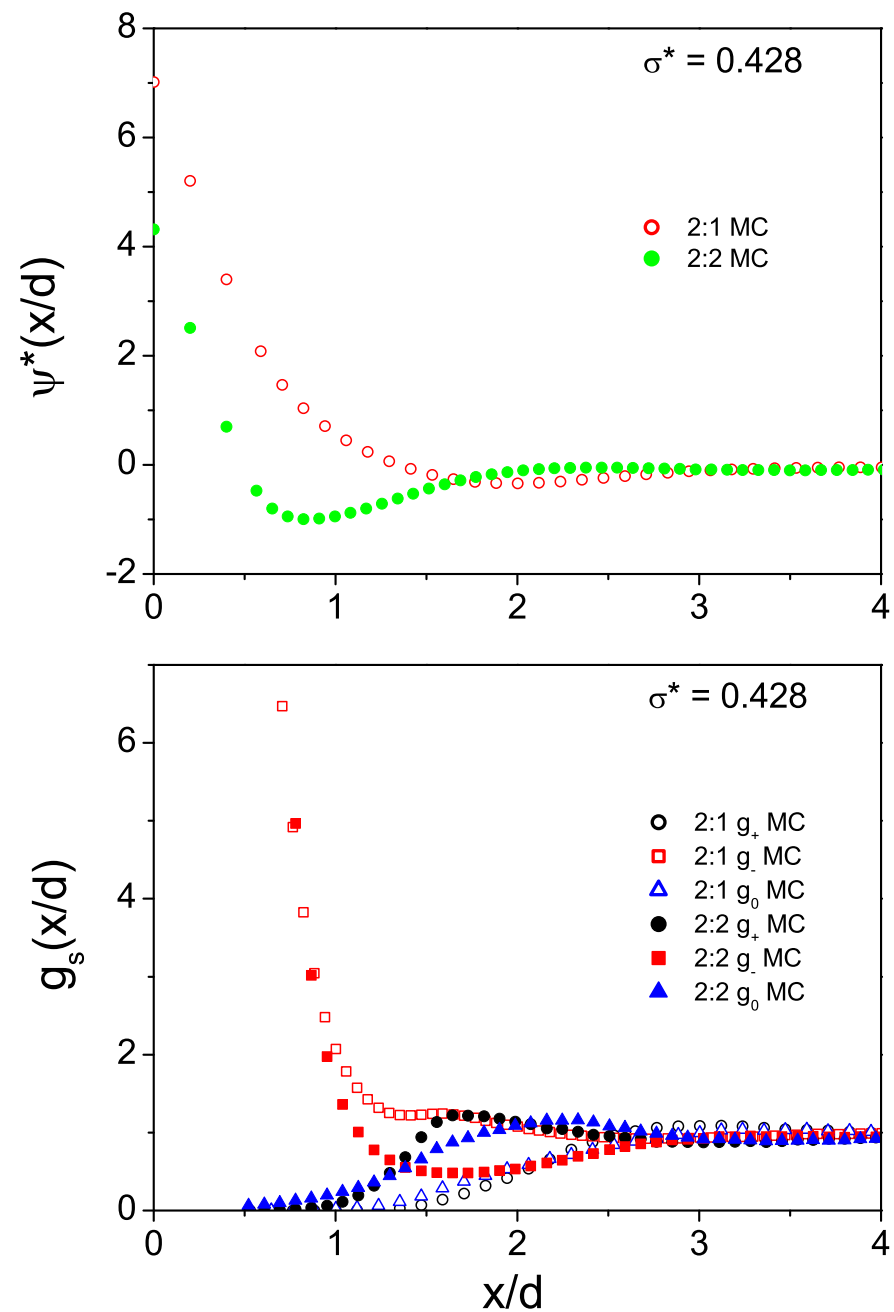

Figure 4

Figure 4: 

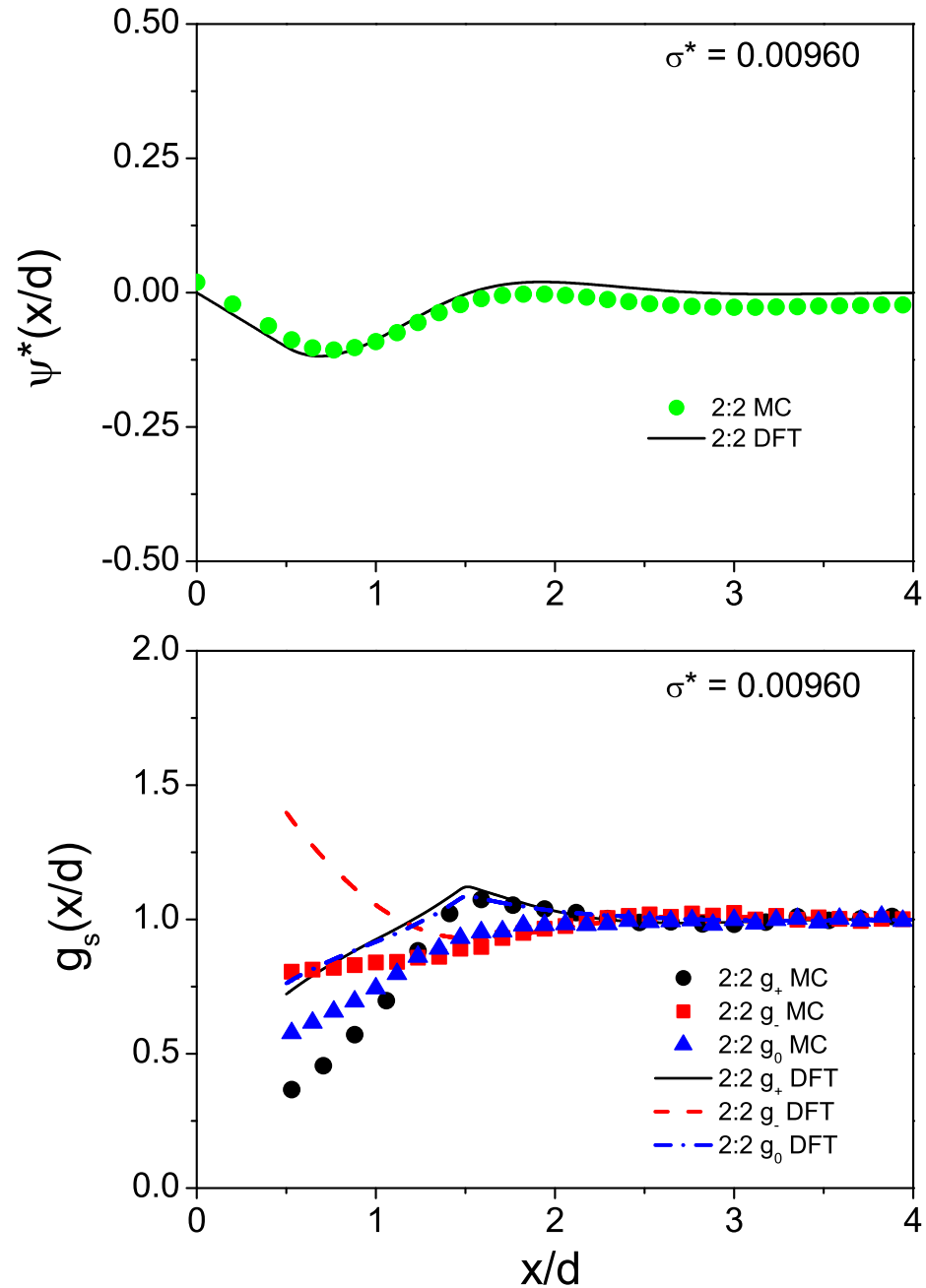

Figure 5

Figure 5: 


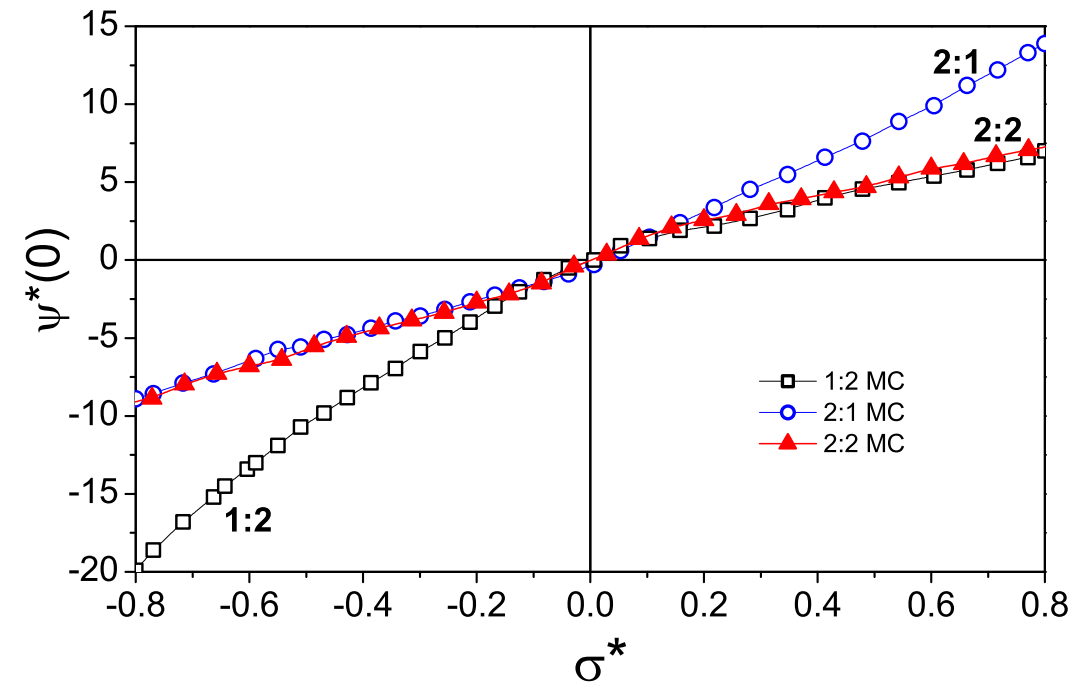

Figure 6

Figure 6: 


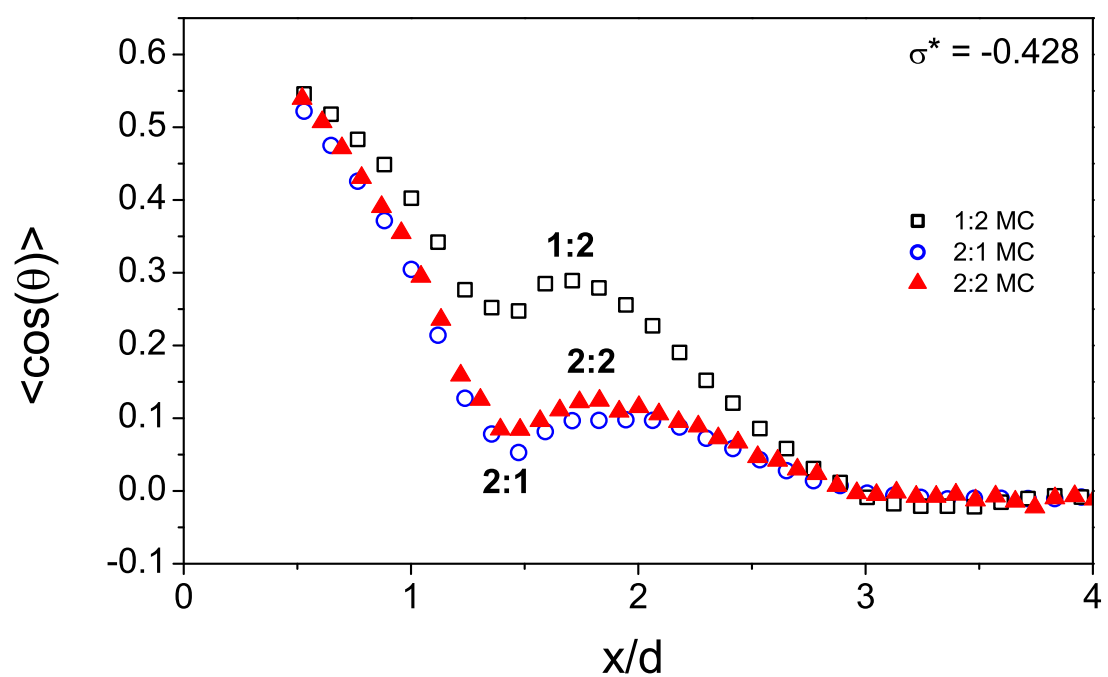

Figure 7

Figure 7: 